\title{
LINEAR MATRIX INEQUALITIES CONTROL DRIVEN FOR NON-IDEAL POWER SOURCE ENERGY HARVESTING
}

\author{
Douglas C. Ferreira \\ UFMT - Federal University of Mato Grosso, Mechanical Engineering Department, Rondonópolis, MT, Brazil \\ e-mail: dcferreira@ufmt.br \\ Fábio R. Chavarette \\ UNESP - Universidade Estadual Paulista, Mathematical Sciences Department, Ilha Solteira, SP, Brazil \\ e-mail: fabioch@mat.feis.unesp.br \\ NeLson J. Peruzzi \\ UNESP - Universidade Estadual Paulista, Mathematical Sciences Department, Jaboticabal, SP, Brazil \\ e-mail:peruzzi@fcav.unesp.br
}

\begin{abstract}
The dynamic model of a linear energy harvester excited by a non-ideal power source is coupled to a controller to maximum vibration adjustment. Numerical analysis is taken to evaluate the energy harvested keeping the vibration optimized for the maximum interaction to the energy source using linear matrix inequalities for control driven. The dimensionless power output, actuation power and net output power is determined. As a result, it is possible to verify that the total energy harvested via exogenous vibration using the proposed controller is increased up to 65 times when in comparison to the open loop system.
\end{abstract}

Keywords: energy harvesting, efficiency, control, non-ideal excitation

\section{Introduction}

Harnessing energy from environment to supply low power devices can be accomplished from sources as thermal gradients, solar radiation and vibration (Huesgen et al., 2008; Cepnik et al., 2011; Miller et al., 2011). Concerning the vibration there are three main groups of harvesters: electrostatic, electromagnetic and piezoelectric (Roundy et al., 2003).

Although harvesting to be a sustainable source of energy, it is still not efficient enough and its use is limited to very little power devices, and enhancing its capacity represents a science frontier. The energy harvesting system main application in the present is for remote sensor nodes, wireless systems and smart structures actuators (Miller et al., 2011; Roundy et al., 2003). To enhance energy harnessed, there are several project solutions focusing in matching the natural frequency according to the vibration source, nevertheless the resonance solution has restrictions for power source and scale.

Regarding the power source, an important consideration is that the environmental vibration has low power and wide range, and normally is random resulting in difficulties for a design to be coincident with the resonance. Considering the scale, it is notable that recent studies concerning the enhancement of energy harvesting efficiency produced better piezoelectric materials (Yeager and Trolier-Mckinstry, 2012; Baek et al., 2011; Kim et al., 2012) and now the designed harvesters are facing new barrier applications of micro sizing. As most power sources from ambient vibration have low frequency, it is difficult to design a small harvester resulting in his natural frequency to be coincident with the resonance. 
Some project solutions regard to tuning a device to the resonance (Challa et al., 2008; Eichhorn et al., 2009) that can be accomplished by mechanical, magnetic and piezoelectric adjustments (Peters et al., 2009; Tang et al., 2013). The mechanical adjustment requires known excitation frequency and high cost energy to tuning, magnetic adjustment has limited tuning result and faces a limited micro sizing requirements, and the piezoelectric adjustment is a promising frontier to enhance energy harvesting capability (Zhu et al., 2010). First studies using piezoelectric adjustment results in negative net output power (Roundy and Zhang, 2005) but latter studies from Zhu et al. (2010) found a mistake in Roundy and Zhang (2005) formulation that not considered mean voltage to active power determination. A piezoelectric tuning solution with positive net output power was numerically and experimentally proven by Lallart and Inman (2010) and Lallart and Guyomar (2010). According to Wang and Inman (2012), the state of the art of enhancement of energy harvesting capability relies in control projects formulation which characterizes the mean objective of this study.

The proposed solution considers that vibration excitation has low energy and the vibration generated by the harvester can influence the source which characterizes a non-ideal power source (Balthazar et al., 2003; Balthazar and Dantas, 2004; Chavarette, 2012). For a limited power in controller systems, the input vibration control influences the own controller which characterizes a non-ideal system (Souza et al., 2008). Thus the model of movement sums the feedback term increasing the number of degrees of freedom (Piccirillo et al., 2008). According to Balthazar et al. (2003), when the vibration source is near natural frequency there appears a jump phenomenon which it is not possible while arriving the resonance as the maximum vibration response.

In this study, the proposed controller is based on Linear Matrix Inequalities (LMI) theory. The dynamic systems control for vibration maximization is performed by optimum control $H \infty$ as a convex optimization problem involving Linear Matrix Inequalities - LMI (Chilali and Gahinet, 1996). The LMI can be in form of linear inequalities, linear convex inequalities or matrix inequalities. Several restriction formats of control theory as Lyapunov and Riccati inequalities can be described via LMI (Antwerp and Braatz, 2000). The LMI has large use in control, mainly because sustains the stability of a system based in restrictions and can incorporated in dynamical systems with not singular parameters that vary within a known range (Wan and Kothare, 2003; Andrea et al., 2008).

To explore controller efficiency, this study is conducted according to a bimorph energy harvester (Erturk and Inman, 2011) and the use of non-ideal power source (Balthazar et al., 2003; Balthazar and Dantas, 2004; Chavarette, 2012). The definition of a Linear Matrix Inequalities controller (LMI) to set up a harvester to optimize interaction to the power source to take advantage of full range of vibration is the main propose of this study.

Considering the paper organization: Section 2 presents the harvester model, Section 3 presents the controller definition, Section 4 presents the efficiency analysis and Section 5 presents the final remarks from this investigation and acknowledgements.

\section{Harvester model}

A non-ideal excitation can be modeled for an unbalanced mass receiving torque by a DC motor as shown in Fig. 1 (Balthazar et al., 2003; Balthazar and Dantas, 2004; Chavarette, 2012).

This arrangement is based in Kononenko model and can be mathematically modeled according to equations (2.1) where $m_{1}$ is DC motor mass, $m_{0}$ is unbalanced mass, $l$ is dumping, $k$ is rigidity, $r$ is the eccentricity distance from the unbalanced mass to the torque source, $\left(I+m_{0} r^{2}\right)$ is moment of inertia of the unbalanced mass, and the state variables are $X$ for the beam tip position and $\varphi$ for the unbalanced mass angular position. The net torque is a function of angular 
velocity $\dot{\varphi}$ and described for $S(\dot{\varphi})=a-b \dot{\varphi}$ (Palácios et al., 2003; Tusset et al., 2013), where $a$ is the net torque applied by the DC motor and $b$ is the resistive net torque constant

$$
\begin{aligned}
& \left(m_{1}+m_{0}\right) \ddot{X}+l \dot{X}+k X=m_{0} r\left(\dot{\varphi}^{2} \cos \varphi+\ddot{\varphi} \sin \varphi\right) \\
& \left(I+m_{0} r^{2}\right) \ddot{\varphi}-m_{0} r \ddot{X} \sin \varphi=S(\dot{\varphi})
\end{aligned}
$$

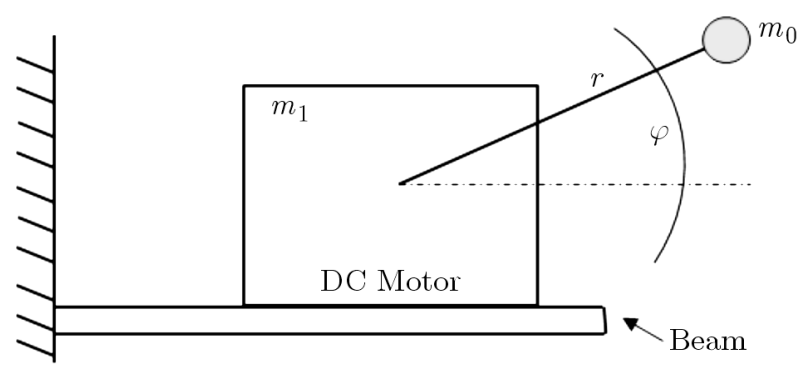

Fig. 1. Non-ideal power source (Chavarette, 2012)

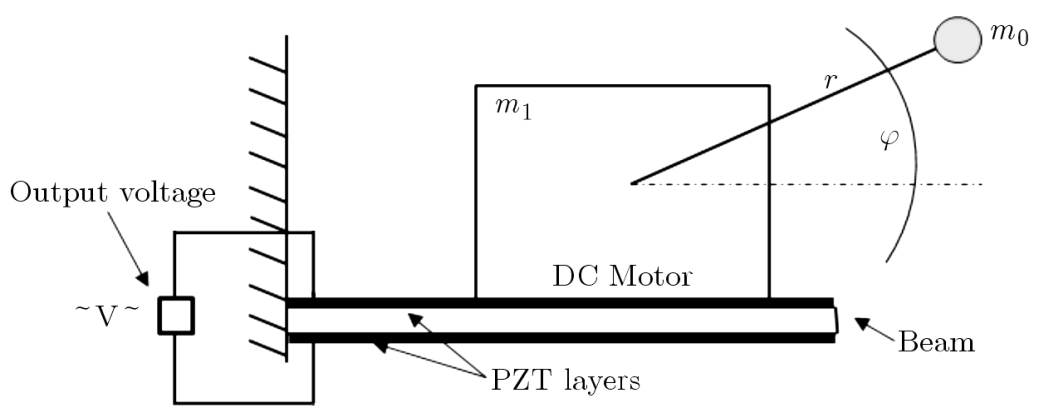

Fig. 2. Energy harvester system (Erturk and Inman, 2011) coupled to non-ideal power source

(Chavarette, 2012)

An energy harvester defined by Erturk and Inman (2011) with two layers of a piezoelectric material and an output voltage was coupled to the non-ideal power source as shown in Fig. 2. It is dimensionlessly modeled according to the resonance as given by equations (2.2), where $\zeta$ is damping factor, $\chi$ is piezoelectric mechanical coupling coefficient, $\Lambda$ is reciprocal of time constant, $\kappa$ is piezoelectric electric coupling coefficient, $\mu$ is unbalanced mass eccentricity, $\xi$ is unbalanced mass eccentricity for moment of inertia, $\alpha$ is the net torque applied by the DC motor and $\beta$ is the resistive net torque constant. The dimensionless state variables are $x$ for tip beam position, $z$ for the angular position of the unbalanced mass and $\nu$ for the output voltage

$$
\begin{aligned}
& \ddot{x}+2 \zeta \dot{x}+\frac{1}{2} x-\chi \nu=\mu\left(\dot{z}^{2} \cos z+\ddot{z} \sin z\right) \\
& \ddot{z}=\xi \ddot{x} \sin z+\alpha-\beta \dot{z} \\
& \dot{\nu}+\Lambda \nu+\kappa \dot{x}=0
\end{aligned}
$$

Isolating $\ddot{x}, \dot{\nu}$ and $\ddot{z}$, equation $(2.2)$ can be presented by

$$
\begin{aligned}
& \ddot{x}=\frac{-\frac{1}{2} x-2 \zeta \dot{x}+\chi \nu+\mu \dot{z}^{2} \cos z+(\alpha-\beta \dot{z}) \mu \sin z}{1-\mu \xi(\sin z)^{2}} \\
& \ddot{z}=\frac{\left(-\frac{1}{2} x-2 \zeta \dot{x}+\chi \nu\right) \xi \sin z+\mu \xi \dot{z}^{2} \cos z \sin z+\alpha-\beta \dot{z}}{1-\mu \xi(\sin z)^{2}} \\
& \dot{\nu}=-\kappa \dot{x}-\Lambda \nu
\end{aligned}
$$


Adopting $x=x_{1}, z=x_{3}$ and $\nu=x_{5}$ and rearranging the terms, the space-state form of equations (2.3) is given by

$$
\begin{aligned}
& \dot{x}_{1}=x_{2} \\
& \dot{x}_{2}=\frac{-\frac{1}{2} x_{1}-2 \zeta x_{2}+\chi x_{5}+\mu x_{4}^{2} \cos x_{3}+\left(\alpha-\beta x_{4}\right) \mu \sin x_{3}}{1-\mu \xi\left(\sin x_{3}\right)^{2}} \\
& \dot{x}_{3}=x_{4} \\
& \dot{x}_{4}=\frac{\left(-\frac{1}{2} x_{1}-2 \zeta x_{2}+\chi x_{5}\right) \xi \sin x_{3}+\mu \xi x_{4}^{2} \cos x_{3} \sin x_{3}+\alpha-\beta x_{4}}{1-\mu \xi\left(\sin x_{3}\right)^{2}} \\
& \dot{x}_{5}=-\kappa x_{2}-\Lambda x_{5}
\end{aligned}
$$

Applying Jacobian, the matrix form of equations (2.4) is given as

$$
\left\{\begin{array}{l}
\dot{x}_{1} \\
\dot{x}_{2} \\
\dot{x}_{3} \\
\dot{x}_{4} \\
\dot{x}_{5}
\end{array}\right\}=\left[\begin{array}{ccccc}
0 & 1 & 0 & 0 & 0 \\
-\frac{1}{2} & -2 \zeta & \mu \alpha & 0 & \chi \\
0 & 0 & 0 & 1 & 0 \\
0 & 0 & -\frac{1}{2} \xi & -\beta & 0 \\
0 & -\kappa & 0 & 0 & -\Lambda
\end{array}\right]\left\{\begin{array}{l}
x_{1} \\
x_{2} \\
x_{3} \\
x_{4} \\
x_{5}
\end{array}\right\}
$$

The main propose of this study is to design a controller to optimize vibration response and, consequently, energy the harvesting performance. In this case, a Linear Matrix Inequalities controller is arranged to maximize the displacement and velocity. As the output voltage has directly influenced by velocity and displacement, it is expected to increase the output voltage and, then, the efficiency.

\section{Controller definition}

To ensure the maximum energy harvested, the controller it is coupled using Linear Matrix Inequalities (LMI) according to Optimum Control $H \infty$. An LMI Control Driven utilizes feedback state to optimize the interaction between exogenous the excitation $w(t)$ and the resulting signal $y(t)$ (Andrea et al., 2008). A sufficient condition for LMI control is the existence of a matrix $\mathbf{X}=\mathbf{X}^{\prime} \in \mathcal{R}^{n \times n}$ and $\mathbf{Y} \in \mathcal{R}^{m \times n}$ satisfying equations

$\min \mu$

$$
\left|\begin{array}{ccc}
\mathbf{A X}+\mathbf{X} \mathbf{A}^{\prime}-\mathbf{B}_{2} \mathbf{Y}-\mathbf{Y}^{\prime} \mathbf{B}_{2}^{\prime} & \mathbf{X C}^{\prime}+\mathbf{Y}^{\prime} \mathbf{D}^{\prime} & \mathbf{B}_{1} \\
\mathbf{C X}+\mathbf{D Y} & -\mathbf{I} & \mathbf{0} \\
\mathbf{B}_{1}^{\prime} & \mathbf{0} & -\mu \mathbf{I}
\end{array}\right|<\mathbf{0}
$$

The space-state model for the proposed controlled system is given by equations (3.2), and explained in a schematic flow chart in Fig. 3

$$
\dot{\mathbf{x}}=\mathbf{A x}+\mathbf{B}_{1} \mathbf{w}+\mathbf{B}_{2} \mathbf{u} \quad \mathbf{y}=\mathbf{C x}
$$

where $\mathbf{A}$ is the state matrix, $\mathbf{B}_{1}$ is the excitation vector, $\mathbf{B}_{2}$ is the feedback state vector and $\mathbf{C}$ is the actuation vector and control signal, in this case, a singular control signal.

The previous study conducted by Erturk and Inman (2011) defined parameters for a bimorph cantilever according to the resonance $\zeta=0.01, \chi=0.05, \kappa=0.5, \Lambda=0.05$, and the studies conducted by Balthazar et al. (2003), Balthazar and Dantas (2004) and Chavarette (2012) 


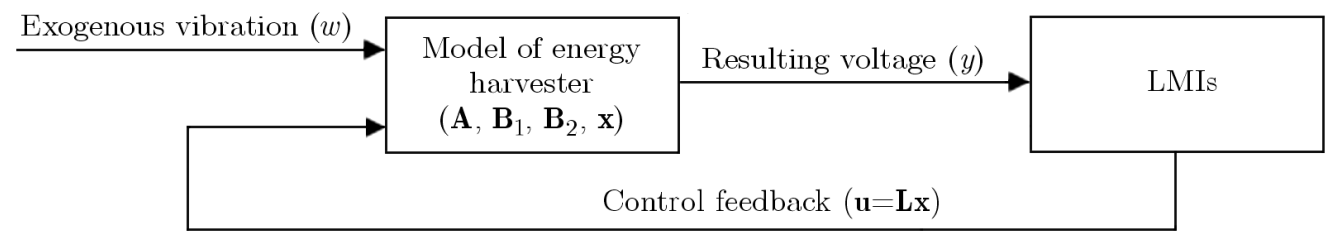

Fig. 3. Dynamic control scheme

defined the parameters for non-ideal excitation $\mu=0.2, \xi=0.3$ and $\beta=1.5$. Replacing the parameters in the state matrix according to equation (2.5) the matrices for equation (3.2) are shown as in the following

$$
\begin{aligned}
& \mathbf{A}=\left[\begin{array}{ccccc}
0 & 1 & 0 & 0 & 0 \\
-0.5 & -0.02 & 0.2 \alpha & 0 & 0.05 \\
0 & 0 & 0 & 1 & 0 \\
0 & 0 & -0.15 & -1.5 & 0 \\
0 & -0.5 & 0 & 0 & -0.05
\end{array}\right] \\
& \left.\mathbf{B}_{1}=\left[\begin{array}{l}
1 \\
1 \\
1 \\
1 \\
1
\end{array}\right] \quad \mathbf{B}\right)_{2}=\left[\begin{array}{l}
1 \\
1 \\
1 \\
1 \\
1
\end{array}\right] \quad \mathbf{C}=\left[\begin{array}{lllll}
1 & 0 & 0 & 0 & 0
\end{array}\right]
\end{aligned}
$$

\section{Efficiency analysis}

For the designed controller the initial parameters are used to excite the beam, and then a LMI controller is set up to the maximum interaction between the excited beam and the external exogenous excitation, in this case, a non-ideal power source. When LMIs are feasible, there is a matrix $\mathbf{X}$ and a feedback matrix $\mathbf{L}$ for the space-state that optimizes the system behavior, given by equation (3.4)

$$
\mathbf{L}=\mathbf{Y X}^{-1}
$$

Once $\mathbf{L}$ is determined, it is possible to determine a feedback space-state matrix for the system $\left(\mathbf{A}_{f}\right)$, given by equation (3.5)

$$
\mathbf{A}_{f}=\mathbf{A}-\mathbf{B}_{2} \mathbf{L}
$$

The variable parameter is the dimensionless net torque applied by the DC motor $\alpha$ that will assume values ranging from 0.4 to 5.0.

\subsection{System response for $\alpha=0.4$}

Considering $\alpha=0.4$ and substituting matrices $\mathbf{A}, \mathbf{B}_{1}, \mathbf{B}_{2}$ and $\mathbf{C}$ given in equations (3.3) in LMIs given in equations (3.1) and solving the inequalities, the resulting feedback matrix $\mathbf{L}$ for a feasible system is

$$
\mathbf{L}=10^{3}\left[\begin{array}{lllll}
6.1648 & 0.0004 & -0.0000 & -0.0007 & -0.0000
\end{array}\right]
$$

Taking into consideration the feedback state matrix $\mathbf{A}_{f}$, it is possible to calculate the feedback parameters, comparing to the original matrix $\mathbf{A}$ in (3.3) as shown in Table 3. 
Table 3. Feedback parameters for $\alpha=0.4$

\begin{tabular}{|c|c|c|c|c|c|c|c|}
\hline $\begin{array}{c}\text { Feedback } \\
\text { parameters }\end{array}$ & $\zeta$ & $\chi$ & $\kappa$ & $\Lambda$ & $\mu$ & $\xi$ & $\beta$ \\
\hline \hline Value & 0.2308 & 0.0749 & 0.9416 & 0.0251 & 0.2988 & 0.2210 & 0.8244 \\
\hline
\end{tabular}

Table 4. System eigenvalues $\left(10^{3}\right)$ for $\alpha=0.4$

\begin{tabular}{|l|l|}
\hline$\lambda_{1}$ & $-6.1635+0.0000 \mathrm{i}$ \\
\hline$\lambda_{2}$ & $-0.0015+0.0000 \mathrm{i}$ \\
\hline$\lambda_{3}$ & $-0.0005+0.0002 \mathrm{i}$ \\
\hline$\lambda_{4}$ & $-0.0005-0.0002 \mathrm{i}$ \\
\hline$\lambda_{5}$ & $-0.0001+0.0000 \mathrm{i}$ \\
\hline
\end{tabular}

The controlled system exhibits stable behavior since all the eigenvalues have the real part negative as shown in Table 4.

Performing Runge-Kutta fourth order algorithm for solving the ordinary differential equations in equation (2.4) for open loop (without control) parameters given in Section 3 and for controlled system parameters given in Table 3, it is possible to visualize the total energy from the system to compare the efficiency from the open loop to the controlled system as shown in Fig. 4. Th following initial conditions are considered $x(0)=1, \dot{x}(0)=0, \nu(0)=0, z(0)=0$ and $\dot{z}(0)=0$ and time samples from 0 to 2500 in the interval of 0.1 totalizing 25000 samples in Figs. 4a-4d and samples from 2000 to 2500 in the interval of 0.1 totalizing 5000 samples in Fig. 4d to exclude transient behavior.

The controlled system presents greater displacements, velocity and output voltage in comparison to the open loop system. To explore control performance, the dimensionless net torque applied by the DC motor assumes the values $\alpha=0.5, \alpha=1.3$ and $\alpha=5.0$, considering parameters below the resonance, at the resonance and beyond it, the feedback vector $\mathbf{L}$ and feedback parameters are given in Table 5.

Table 5. Feedback parameters $\alpha=0.5, \alpha=1.3$ and $\alpha=4.0$

\begin{tabular}{|c|c|c|}
\hline$\alpha$ & $\mathbf{L}\left(10^{3}\right)$ & Feedback parameters \\
\hline 0.5 & {$[4.7795,0.0004,-0.0000,-0.0007,-0.0000]$} & $\begin{aligned} \zeta=0.2304, \chi & =0.0742, \kappa=0.9408 \\
\Lambda=0.0258, \mu & =0.2689, \xi=0.2311 \\
\beta & =0.8449\end{aligned}$ \\
\hline 1.3 & {$[2.2552,0.0005,-0.0000,-0.0007,-0.0000]$} & $\begin{aligned} \zeta=0.2537, \chi & =0.0691, \kappa=0.9873 \\
\Lambda=0.0309, \mu & =0.2173, \xi=0.2551 \\
\beta & =0.8212\end{aligned}$ \\
\hline 5.0 & {$[2.4482,0.0006,0.0000,-0.0007,-0.0000]$} & $\begin{array}{c}\zeta=0.3096, \chi=0.0639, \kappa=1.0991 \\
\Lambda=0.0361, \mu=0.1957, \xi=0.3425 \\
\beta=0.8231\end{array}$ \\
\hline
\end{tabular}

Making use of the Runge-Kutta fourth order method for solving ordinary differential equations in (2.4) for open loop (without control) parameters given in Section 3 and for the controlled system parameters given in Table 5 we find analogous results for the initial conditions $x(0)=1$, $\dot{x}(0)=0, \nu(0)=0, z(0)=0$ and $\dot{z}(0)=0$ and time samples from 0 to 2500 in the interval of 0.1 totalizing 25000 samples in Figs. 5a, 5c, 5e and samples from 2000 to 2500 in the interval of 0.1 totalizing 5000 samples to exclude transient behavior in Figs. 5b, 5d, $5 \mathrm{f}$.

It is possible to verify that the system energy is greater for the control system when out of the resonance for $\alpha=0.5$ and $\alpha=5.0$. When the system is at the resonant behavior at $\alpha=1.3$, 

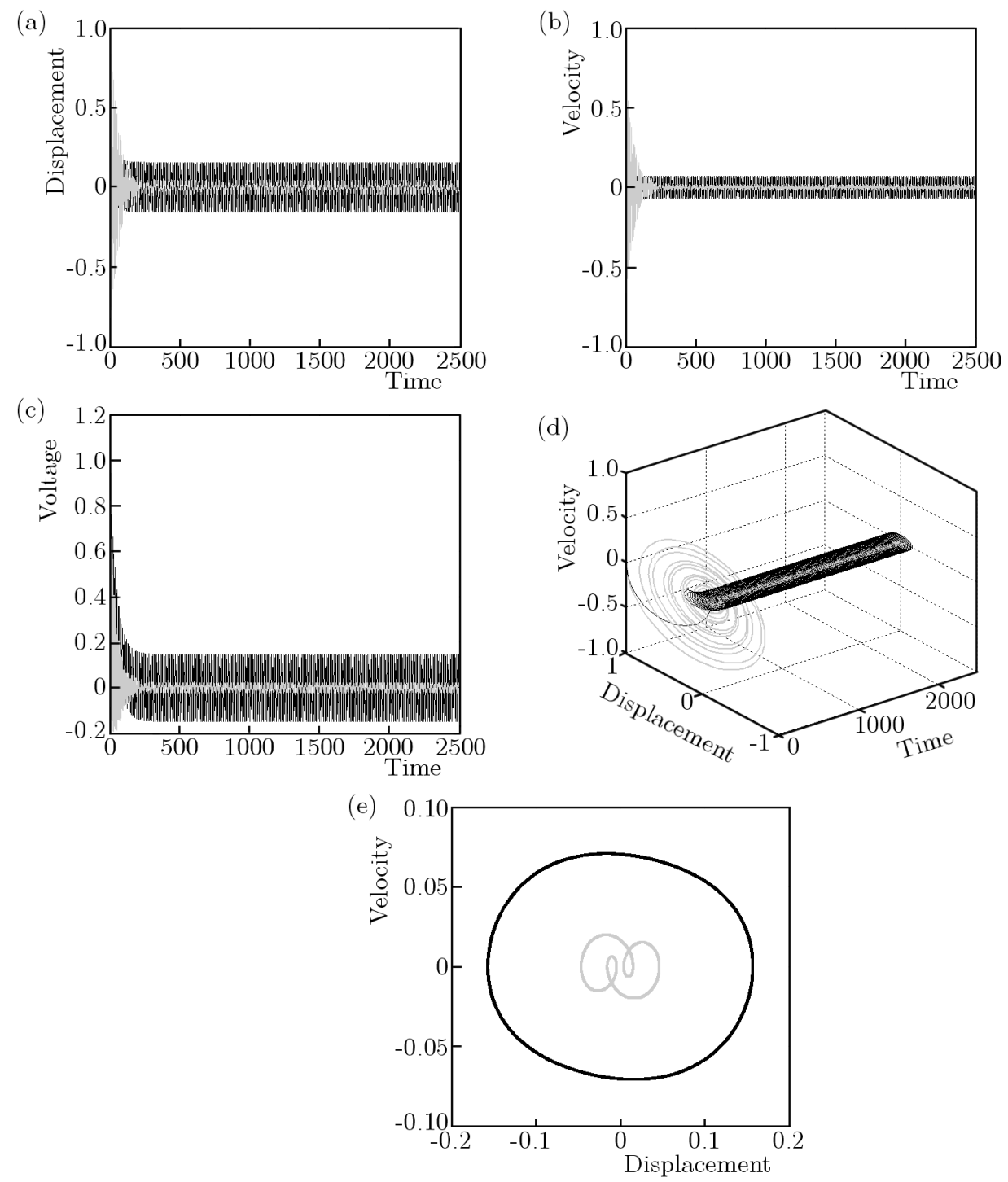

Fig. 4. System behavior for $\alpha=0.4$ (open loop system - grey line, controlled system - black line); (a) displacement, (b) velocity, (c) output voltage, (d) phase portrait time sample and (e) phase portrait without transient

it is not possible for the controller to get more energy than the resonance, and the controlled system gives less energy than the open loop system.

According Tang and Zuo (2011), the system output power $(P)$ is given by equation (4.3) related to the output voltage root mean square $\left(V_{r m s}\right)$ squared, divided by the load resistance $(R)$

$$
P=\frac{V_{r m s}^{2}}{R}
$$

As the model is dimensionless, a dimensionless output power $(\Phi)$ given for the root mean square of the dimensionless output voltage $\left(\nu_{r m s}\right)$ squared, divided by the dimensionless load resistance $(\Psi)$, as shown in (4.4), is considered

$$
\Phi=\frac{\nu_{r m s}^{2}}{\Psi}
$$

For the propose of numerical simulation, a value of the load resistance $\Psi=0.1$ and for $\alpha$ ranging from 0.5 to 5.0 the dimensionless output power $\Phi$ values given in Table 6 are assumed. 

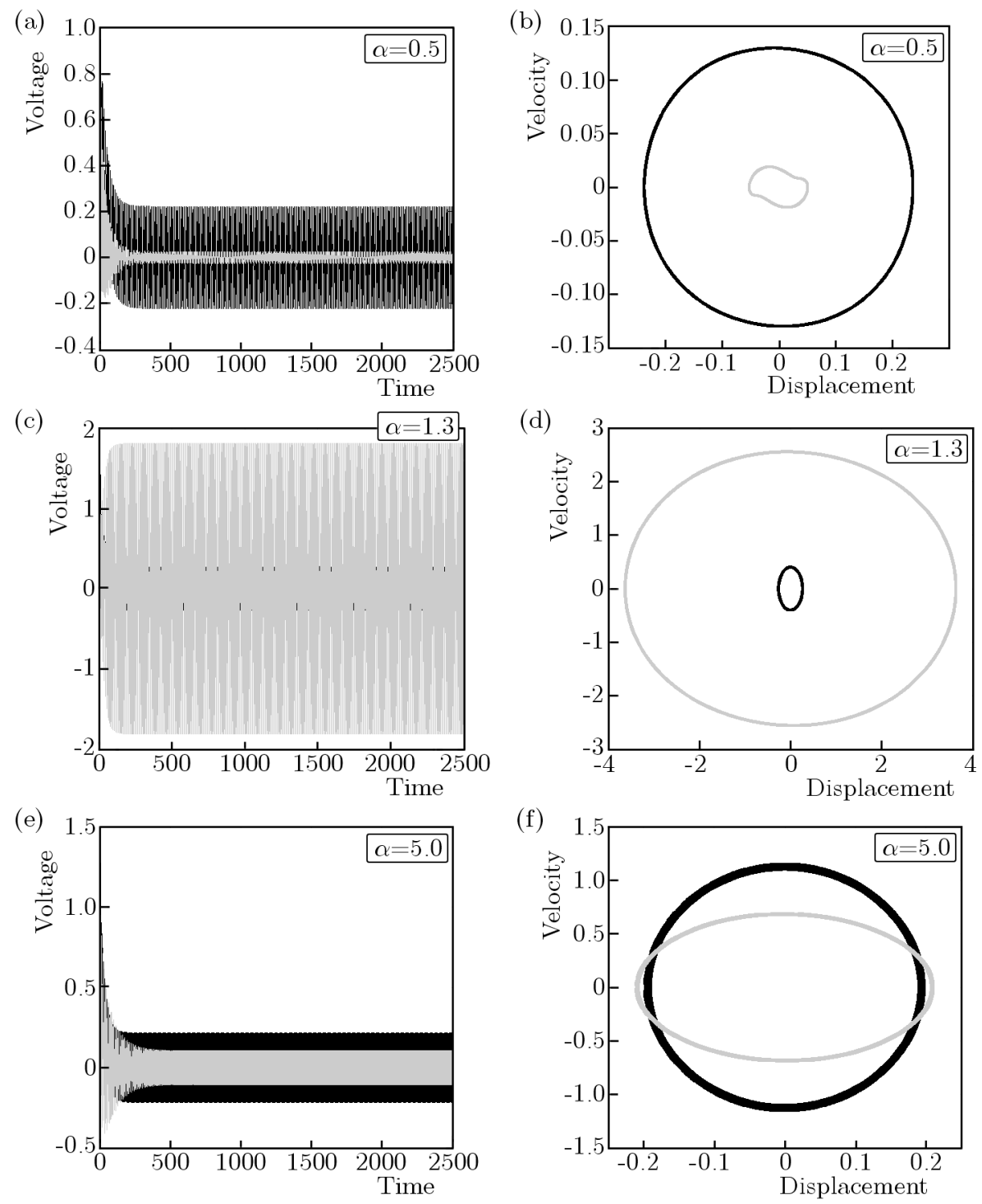

Fig. 5. System behavior (open loop system - grey line, controlled system - black line); (a), (c), (e) output voltage, (b), (d), (f) phase portrait without transient

Table 6. Dimensionless power $\Phi$ for $\alpha$ ranging from 0.4 to 5.0

\begin{tabular}{|c|c|c|}
\hline Net torque $(\alpha)$ & $\Phi-$ Open loop & $\Phi$ - Controlled \\
\hline \hline 0.4 & 0.0247 & 0.1695 \\
\hline 0.5 & 0.0250 & 0.3040 \\
\hline 1.3 & 16.0565 & 0.4015 \\
\hline 5.0 & 0.0953 & 0.2995 \\
\hline
\end{tabular}

Expanding the investigation for other values of the net torque $(\alpha)$, the resulting dimensionless power $(\Phi)$ is given in Fig. 6.

The LMI control show the efficiency of enhancing the system dimensionless output power as shown in Fig. 6 and not considering the resonance effect where the dimensionless output power is greater in the open loop system. This fact is explained because the higher energy orbit available in the system is the resonance behavior. To better visualize the control efficiency, the resonance net torque is shown in Fig. 7. 


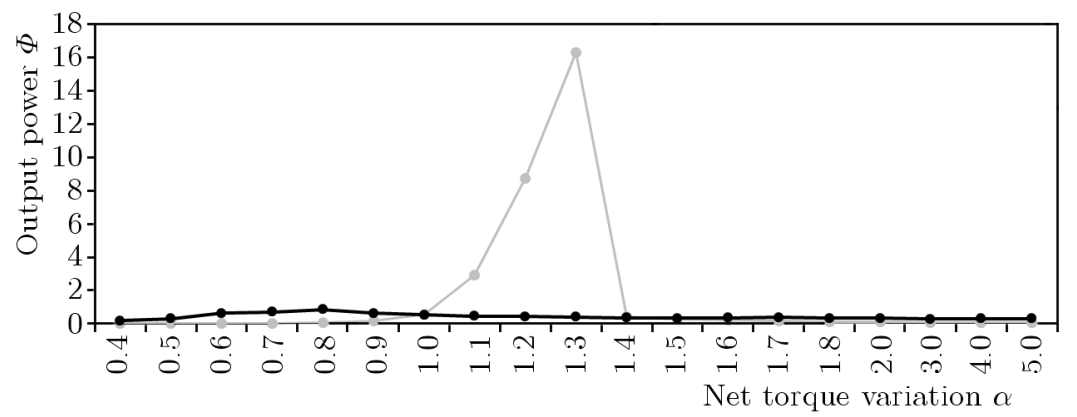

Fig. 6. Dimensionless output power $(\Phi)$ of the open loop compared to the controlled system; open loop system - grey line, controlled system - black line

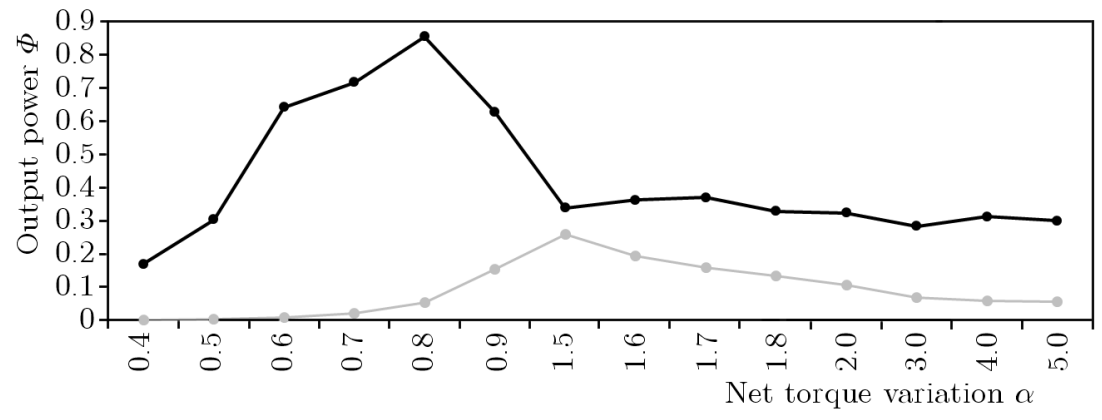

Fig. 7. Dimensionless output power $(\Phi)$ of the open loop compared to the controlled system; open loop system - grey line, controlled system - black line. Suppressing the resonance behavior

To evaluate the dimensionless net output power $\left(\Phi_{\text {net }}\right)$, Roundy and Zang (2005) compared the frequency of the open loop system to the controlled system, and later Lallart and Inman (2010) used the same evaluation to compare the output power from the open loop and controlled systems. In both cases, the power used for control (action power) is referred to the amount of change of the system parameters, in other words, it is the energetic cost to change the system behavior. Based on experimentally proven evaluations, the action power $\left(\Phi_{a c t}\right)$ is determined by applying the Runge-Kutta fourth order method to solve the ordinary differential equations in (2.4) for the absolute difference of the open loop and controlled parameters of each net torque $(\alpha)$. The results are shown in Fig. 8 .

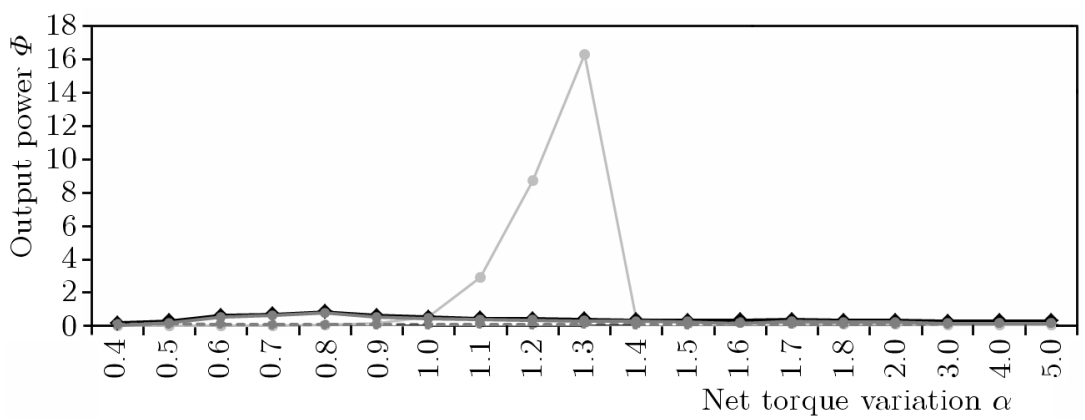

Fig. 8. Output power for the open loop system (grey line); output power for the controlled system (black line); actuation power (dotted line); net output power (solid dark grey line)

To better visualize the control efficiency, the resonance net torque is depicted in Fig. 9. The summary of power gains resulting from the control is shown in Table 7. 


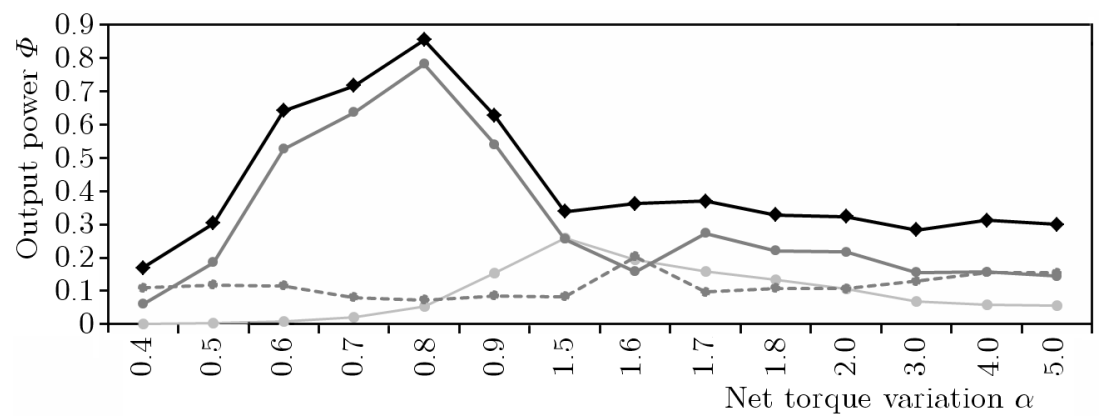

Fig. 9. Output power for the open loop system (grey line); output power for the controlled system (black line); actuation power (dotted line); net output power (solid dark grey line). Suppressing the resonance behavior

Table 7. Summary of dimensionless net power gains for the controlled system

\begin{tabular}{|c|c|c|c|c|c|c|c|c|c|c|}
\hline$\alpha$ & 0.4 & 0.5 & 0.6 & 0.7 & 0.8 & 0.9 & 1.0 & 1.1 & 1.2 & 1.3 \\
\hline Net power gain & 40.69 & 59.67 & 65.85 & 31.70 & 14.99 & 3.54 & 0.77 & 0.13 & 0.04 & 0.02 \\
\hline$\alpha$ & 1.4 & 1.5 & 1.6 & 1.7 & 1.8 & 2.0 & 3.0 & 4.0 & 5.0 & \\
\hline Net power gain & 0.68 & 0.99 & 0.82 & 1.74 & 1.64 & 2.03 & 2.28 & 2.65 & 2.59 & \\
\hline
\end{tabular}

\section{Final remarks}

Various studies regarding the energy harvesting have been carried out for the last few years to enhance the capability of the energy harvesters. In the same direction, this investigation presents a study of control of the dynamic behavior based on Linear Matrix Inequalities Optimum $H \infty$ method and a non-ideal excitation as the power source. As the main result, it has been possible to verify a significant increase in the system energy available for harvesting when applying a controller to the system. Velocity and displacement have direct effect on the output voltage and, consequently, on the output power for energy harvesting. The proposed controller is able to increase the net output power up to 65 times. Table 8 shows the comparison of other studies with the present research.

Table 8. Comparison of the obtained results with other works

\begin{tabular}{|l|c|}
\hline \multicolumn{1}{|c|}{ Author } & $\begin{array}{c}\text { Resulting frequency } \\
\text { band }[\mathrm{Hz}]\end{array}$ \\
\hline \hline Roundy and Zhang (2005) & $61.8-67.0$ \\
\hline Wu et al. (2006) & $91.0-94.5$ \\
\hline Peters et al. $(2009)$ & $66.0-89.0$ \\
\hline Lallart and Inman (2010) & $8.1-112.0$ \\
\hline This work & all the band \\
\hline
\end{tabular}

Whereas other energy harvesting methods, such as piezoelectric tuning, increase the resonant band, this control approach enhances energy not regarding the band but focusing on the output power optimization with considerable efficiency for small power excitations.

In this study, the parameters are considered constant, however in a real situation, damping and electrical coupling parameters depend on environmental conditions, for example humidity and temperature. Such variation in the parameters results in polytopic uncertainties. Thus, instead of an optimum method, a robust LMI method is required (Andrea et al., 2008) which characterizes possible research continuation. The energy sink due to controller circuit is result 
of the electronic architecture and is not explored in this study as another possible research exploration area.

Acknowledgments

The authors would like to thank CNPq (Proc. No. 301769/2012-5) and Federal University of Mato Grosso (UFMT) for funding this research.

\section{References}

1. Andrea C.Q., Pinto J.O.P., Assunção E., Teixeira M.C.M., Galotto L.J., 2008, Optimum control $H \infty$ of non-linear systems with fuzzy Takagi-Sugeno models (in Portuguese), Controle and Automação, 19, 3

2. Antwerp J.G., Brattz R.D., 2000, A tutorial on linear and bilinear matrix inequalities, Journal of Process Control, 10, 363-385

3. Baek S.H., Park J., Kim D.M., Aksyuk V.A., Das R.R., Bu S.D., Felker D.A., et al., 2011, Giant piezoelectricity on Si for hyperactive MEMS, Science, 334

4. Balthazar J.M., Dantas M.J.H., 2004, On local analysis of oscillations of a non-ideal and non-linear mechanical model, Meccanica, 39, 4, 313-330

5. Balthazar J.M., Mook D.T., Weber H.I., Brasil R.M.L.R.F., Fenili A., Belato D., FELIX P.J.L., 2003, An overview on non-ideal vibrations, Meccanica, 38, 613-621

6. Cepnik C., Radler O., Rosenbaum S., Ströhla T., Wallrabe U., 2011, Effective optimization of electromagnetic energy harvesters through direct computation of the electromagnetic coupling, Sensors and Actuators, 167, 416-421

7. Challa V.R., Prasad M.G., Shi Y., Fisher F.T., 2008, A vibration energy harvesting device with bidirectional resonance frequency tenability, Smart Materials and Structures, 17

8. Chavarette F.R., 2012, On an optimal linear control of a chaotic non-ideal Duffing system, Advances in Mechanical Engineering, 2, 3

9. Chilali M., Gahinet P., 1996, $H \infty$ design with pole placement constrains: an LMI approach, IEEE Transactions on Automatic Control, 41, 3

10. Eichhorn C., Goldschmidtboeing F., Porro Y., Woins P., 2009, A piezoelectric harvester with an integrated frequency tuning mechanism, PowerMEMS, Washington DC, USA, December $1-4$

11. ERturk A., Inman D., 2011, Broadband piezoelectric power generation on high-energy orbits of the bistable Duffing oscillator with electromechanical coupling, Journal of Sound and Vibration, 330, 2339-2353

12. Huesgen T., Woias P., Kockmann N., 2008, Design and fabrication of MEMS thermoelectric generators with high temperature efficiency, Sensors and Actuators A, 423-429

13. Kim S., Leung A., Koo C.Y., Kuhn L., Jiang W., Kim D., Kingon A.I., 2012, Leadfree $(\mathrm{Na} 0.5 \mathrm{~K} 0.5)(\mathrm{Nb} 0.95 \mathrm{Ta} 0.05) \mathrm{O} 3-\mathrm{BiFeO} 3$ thin films for MEMS piezoelectric vibration energy harvesting devices, Materials Letters, 69, 24-26

14. Lallart M., Guyomar D., 2010, Piezoelectric conversion and energy harvesting enhancement by initial energy injection, Applied Physics Letters, 97

15. Lallart M., Inman D.J., 2010, Frequency self-tuning scheme for broadband vibration energy harvesting, Journal of Intelligent Material Systems and Structures, 21, 897-906

16. Miller L.M., Halvorsen E., Dong T., Wright P.K., 2011, Modeling and experimental verification of low-frequency MEMS energy harvesting from ambient vibrations, Journal of Micromechanics and Microengineering, 21, 045029, pp. 13 
17. Palácios J.L., Balthazar J.M., Brasil R.M.L.F.R., 2003, A short note on a nonlinear system vibrations under two non-ideal excitations, Journal of the Brazilian Society of Mechanical Sciences and Engineering, XXV, 391-395

18. Peters C., Maurath D., Schock W., Mezger F., Manoli Y., 2009, A closed-loop widerange tunable mechanical resonator for energy harvesting systems, Journal of Micromechanics and Microengineering, 19, pp. 9

19. Piccirillo V., Balthazar J.M., Pontes Jr. B.R., Felix J.L.P., 2008, On a nonlinear and chaotic non-ideal vibrating system with shape memory alloy (SMA), Journal Of Theoretical and Applied Mechanics, 46, 597-620

20. Roundy S., Wright P.K., Rabaey J., 2003, A study of low level vibrations as a power source for wireless sensor nodes, Computer Communications, 26, 1131-1144

21. Roundy S., Zhang Y., 2005, Toward self-tuning adaptive vibration-based microgenerators, Proceedings of Smart Structures, Devices and Systems, 5649, 373-384

22. Souza S.L.T., Caldas I.L., Viana R.L., Balthazar J.M., 2008, control and chaos for vibroimpact and non-ideal oscillators, Journal of Theoretical and Applied Mechanics, 46, 641-664

23. TAng L., Yaowen Y., Soh C.K., 2013, Broadband vibration energy harvesting techniques, [In:] Advances in Energy Harvesting Methods (Chapter Two), N. Elvin and A. Erturk (Eds.), Springer Science \& Business Media, New York

24. TANG X., Zuo L., 2011, Enhanced vibration energy harvesting using dual-mass systems, Journal of Sound and Vibration, 330, 5199-5209

25. Tusset A.M., Balthazar J.M., Felix J.L.P., 2013, On elimination of chaotic behavior in a non-ideal portal frame structural system, using both passive and active controls, Journal of Vibration and Control, 19, 6, 803-813

26. Wan Z., Kothare M.V., 2003, An efficient off-line formulation of robust model predictive control using linear matrix inequalities, Automatica, 39, 837-846

27. WANG Y., InMAn J.D., 2012, A survey of control strategies for simultaneous vibration suppression and energy harvesting via piezoceramics, Journal of Intelligent Material Systems and Structures, 23, 18, 2021-2037

28. Wu W.-J., Chen Y.-Y., Lee B.-S., He J.-J., Peng Y.-T., 2006, Tunable resonant frequency power harvesting devices, Proceedings of Smart Structures and Materials, 6169, 55-62

29. Yeager C.B., Trolier-Mckinstry S., 2012, Epitaxial Pb(Zrx,Ti1-x)O3 (0.30?x?0.63) films on (100) $\mathrm{MgO}$ substrates for energy harvesting applications, Journal of Applied Physics, 112

30. Zhu, D., Tudor, M. J., Beeby, S. P., 2010, Strategies for increasing the operating frequency range of vibration energy harvesters: a review, Measurement Science and Technology, 21, pp. 29 